\title{
Utility of Fine Needle Aspiration for Diagnosis of Plasmacytoma
}

\author{
Nitya Prabhakaran ${ }^{\mathrm{a}, \mathrm{c}}$, Alexander W. Scott ${ }^{\mathrm{b}}$, Magda Esebua ${ }^{\mathrm{a}}$
}

\begin{abstract}
Plasmacytomas are rare extramedullary plasma cell neoplasms that present in bone and/or soft tissue. Diagnosis can require a variety of laboratory approaches such as serum protein electrophoresis, bone marrow biopsy, Bence Jones protein assays, and imaging. However, few case reports describe using fine needle aspiration for diagnosis. We present a case of a plasmacytoma diagnosis in a 55-year-old patient, who initially presented to the emergency room with symptoms of chest pain. Imaging revealed right apical and medial lung mass extending just posterior to the trachea with invasion and destruction of the anterolateral T3 vertebral body. Multiple smaller hypodensities were also present in thoracic vertebral bodies, ribs, and sternum. Biopsies were performed on the mediastinal mass. Fine needle aspiration revealed groups of mature and immature plasma cells at various stages of maturation. Flow cytometry demonstrated a kappa light chain-restricted plasma cell population. Paraffin immunoperoxidase studies showed that the neoplastic cells bore monotypic kappa light chains. The patient's presentation emphasizes the utility of fine needle aspiration, in conjunction with other ancillary studies, for diagnosing plasmacytomas.
\end{abstract}

Keywords: Fine needle aspiration; Plasmacytoma; Plasma cells

\section{Introduction}

Extramedullary plasmacytoma (EMP) has been defined as an autonomous proliferation of monoclonal plasma cells with varying degrees of differentiation, producing a circumscribed mass or diffuse infiltration [1-3]. The most common sites of EMP are the head, neck, lymph nodes and skin, but it can also be found at various other sites such as bone, nasal cavities, oral cavity, breast, soft tissue, testis and thyroid $[1,2,4-8]$. This is a rare plasma cell neoplasm, constituting approximately $3-6 \%$

Manuscript submitted November 30, 2018, accepted December 10, 2018

aDepartment of Pathology and Anatomical Sciences, University of Missouri, Columbia, MO, USA

${ }^{b}$ Washington University School of Medicine in St.Louis, St. Louis, MO, USA ${ }^{\mathrm{c} C}$ Corresponding Author: Nitya Prabhakaran, Department of Pathology and Anatomical Sciences, Pathology and Anatomic Sciences, University of Missouri School of Medicine, One Hospital Drive, Columbia, MO 65212, USA. Email: prabhakarann@health.missouri.edu

doi: https://doi.org/10.14740/jmc3223 of all plasma cell neoplasms [1, 3, 4, 5, 7-12].

EMPs are rare tumors and can be mistaken for lymphomas or carcinomas depending on the location $[2,13]$. This malady affects most patients in the sixth decade of life. These are typically discovered when they cause compressive symptoms such as dysphagia [12]. Very few reports about EMP of the mediastinum are available in the literature. These disorders represent a spectrum of tumor aggressiveness, from the rather indolent primary extramedullary plasmacytoma to rapidly progressive multiple myeloma [13].

Fine needle aspiration (FNA) is a simple, rapid and inexpensive procedure widely used in the assessment of a variety of lesions involving the breast, lymph nodes, thyroid, salivary glands, and skin with a high sensitivity and specificity [14]. However, there is a dearth of information concerning the use of FNA in the diagnosis of extra-nodal and extramedullary hematopoietic malignancies [14]. Here, we discuss the case of our patient who was diagnosed with extramedullary plasmacytoma arising in the posterior mediastinum by FNA. Through this case report we attempt to highlight the utility of FNA in extramedullary hematopoietic neoplasms.

\section{Case Report}

A 55-year-old male patient with a previous history of cardiac catheterization, hypertension, and vitamin D deficiency was admitted to the University Hospital Emergency Department for chest pain. Imaging (Fig.1) for possible pulmonary embolism

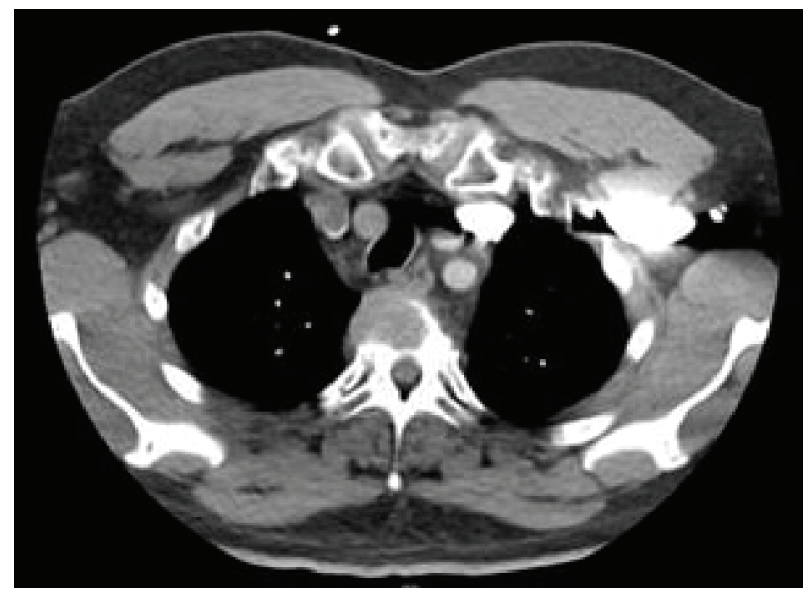

Figure 1. A large mediastinal mass with multiple hypodensities. 


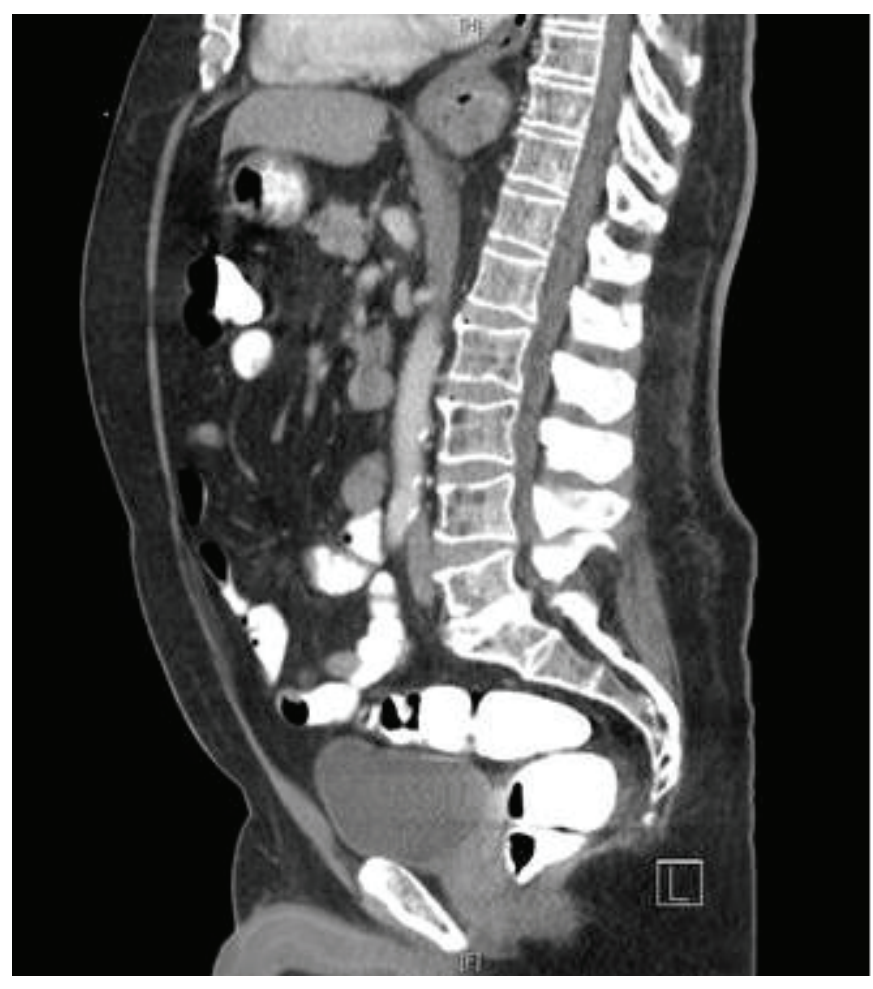

Figure 2. Multiple lytic bone lesions along the spine.

demonstrated right apical and medial lung mass extending just posterior to the trachea with invasion and destruction of the anterolateral T3 vertebral body. Multiple smaller hypodensities were also noted in the thoracic vertebral bodies, ribs, and sternum. Multiple lytic lesions were present along the spine (Fig. 2). The differential diagnosis at the time was multiple myeloma versus metastatic disease versus posterior mediastinal tumors of ganglioma, neuroma, and lymphoma.

Other investigations included IgG serum, total proteinelectrophoresis, alpha-1, alpha-2, IFE beta, IFE gamma, and serum immunoglobulin A levels which were all within normal limits. Serum immunoglobulin $\mathrm{M}$ was low. Carcinoembryonic antigen (CEA), lactate dehydrogenase $\mathrm{LDH}$, and alphafetoprotein (AFP) were all negative. The patient likewise had negative antibodies for Histoplasma, Blastomyces, Aspergil- lus, or Coccidoides. Since the above serum markers had failed to provide a definitive diagnosis, the patient underwent biopsy with endoscopic ultrasound. It was determined that the mediastinal mass and adjacent lymph nodes, rather than the bony lesions, would be easier to biopsy. The mass and lymph node were sampled in the usual manner. The aspirated lymph node sample was negative for malignancy.

Approval was obtained from the institutional IRB at the University of Missouri Hospitals and Clinics, Columbia, Missouri. Fine needle aspiration was prepared by standard methods of thin preps and cell blocks. Immunostaining was performed following the standard protocol on a Dako Immunostainer (Dako, Carpinteria CA). Flow cytometric immunophenotyping was performed on a FacsCanto II flow cytometer (BectonDickinson, Franklin Lakes, NJ) using in-house 8-color protocols. The sample was tested against the following antibodies: CD15, CD34, CD33, CD13, CD11B, HLADR, CD16, CD45, Kappa, Lambda, CD5, CD19, CD23, CD20, CD10, CD4, CD8, CD2, CD56, CD3, CD7, cKappa, cLambda, CD38, and CD138.

\section{Results}

IgG serum, total protein electrophoresis, alpha-1; alpha-2, IFE beta, IFE gamma, and serum immunoglobulin A were all within normal limits. Serum immunoglobulin $\mathrm{M}$ was low. A FNA of the mass and lymph node was performed. The aspirated lymph node sample was negative for malignancy. Flow cytometry (Fig. 3) showed a kappa light chain-restricted plasma cell population. These plasma cells demonstrated bright CD38 and CD138, moderate to bright positive CD56 and CD200, and dim positive for CD45 and CD123.

Microscopic analysis demonstrated that the aspirated sample from the mediastinal mass showed groups of mature and immature plasma cells at various stages of maturation displaying eccentrically placed nuclei and perinuclear hofs (Figs. 4$6)$.

\section{Discussion}

Dalrymple and Bence-Jones first identified a plasma cell dys-
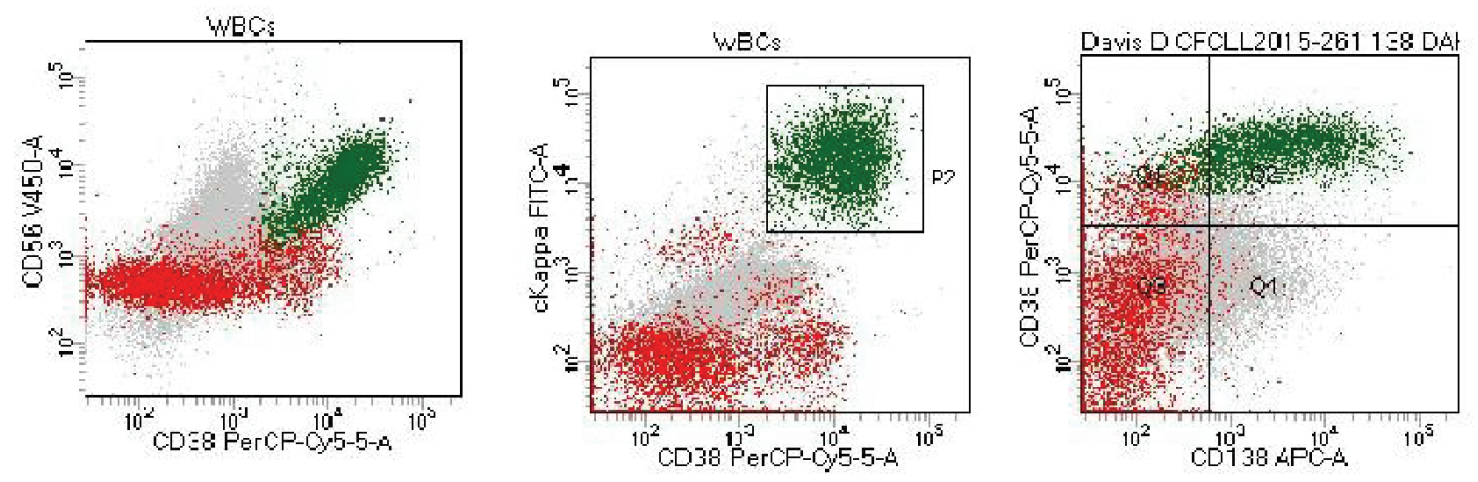

Figure 3. The neoplastic cells stain moderate to bright CD200, bright CD56 and CD38, and show kappa light chain restriction. 


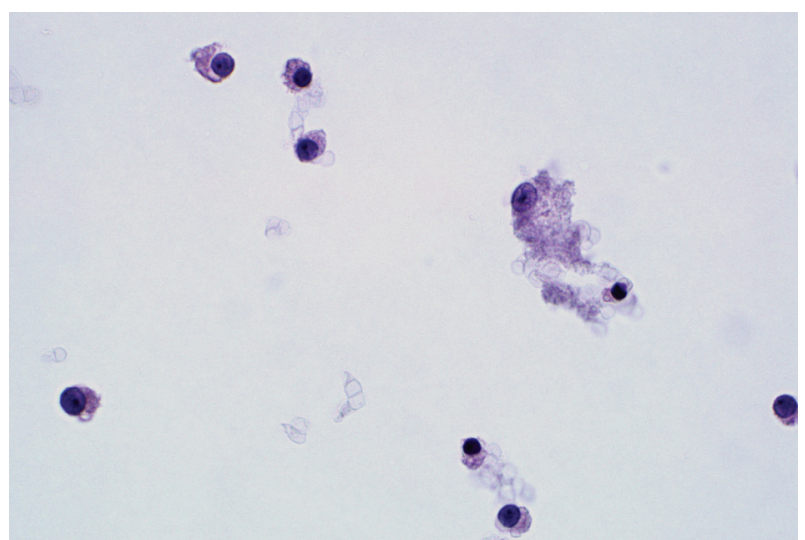

Figure 4. A thin preparation stained by Papanicolaou stain highlights neoplastic plasma cells with Russell body $(60 \times)$.

crasia in 1846 when they described a condition with diffuse bone pain and proteinuria. However, it was not until 1873 that Rustizky et al successfully recognized this condition as a distinct histopathologic entity: multiple myeloma [11]. The first report of EMP was published in 1905. Since then 869 cases of EMPs have been described in the literature. However, only a few cases of EMP diagnosed by FNA have been reported $[1,7]$. Majority of the EMPs, over $80 \%$ were located in the aerodigestive tract $[3,7,10]$. Pulmonary or pleural EMPs were only seen in 12 of those cases [7, 10]. EMP arising in the posterior mediastinum is an extremely rare event and very few case reports are available in the literature $[3,11]$. There are other case reports of EMP arising in the tonsils, larynx, trachea, thyroid and breast, liver and gall bladder (Table 1, [1, $4,6,9,12])$.

After the diagnosis of EMP, the patient underwent a bone marrow aspiration and biopsy procedure which confirmed the presence of clonal plasma cells in the bone marrow and a diagnosis of plasma cell neoplasm consistent with multiple myeloma was made. While the prognosis of EMP is excellent, multiple myeloma carries a grim prognosis. However, even if

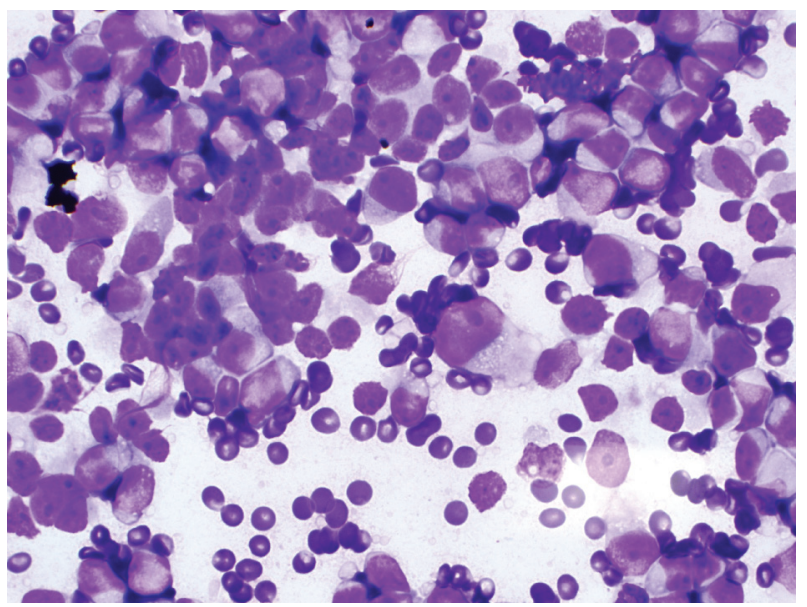

Figure 5. A highly cellular aspirate stained with Diff-Quick stain demonstrates immature cells with blue cytoplasm and perinuclear hof with fine chromatin and prominent nucleoli $(60 \times)$.

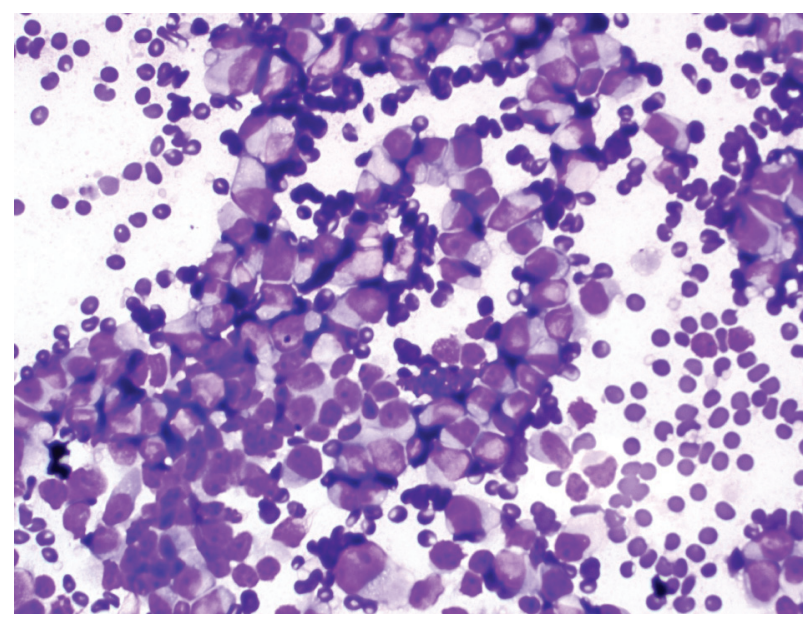

Figure 6. A thin preparation stained with Diff-Quick stain demonstrates immature plasma cells at various stages of maturation $(60 \times)$.

the prognosis of EMP is relatively favorable, a local recurrence or a progression to multiple myeloma has been described in up to $20 \%$ of the cases $[5,8]$.

There is often a lack of distinguishing clinical and radiological features of EMP. Thus the final impression is based on a pathological diagnosis. Usually plasma cell neoplasms demonstrate neoplastic monoclonal plasma cells in varying stages of maturation $[3,5,6,9,10]$. They also demonstrate light chain restriction which can be kappa or lambda. In the case of our patient there was kappa light chain restriction. In addition, EMP should be differentiated from other types of lymphoma (marginal zone, immunoblastic and mucosa-associated lymphoid tissue lymphoma) [10].

The success of a FNA diagnosis is dependent on an adequate yield of material for evaluation that is usually provided by the clinician or radiologist. It also involves a multidisciplinary approach including collaboration and communication between pathologists to reach a consensus diagnosis that is firmly supported by diagnostic information. This approach requires understanding of the limitations of immunophenotyping by flow cytometry, and understanding of the differential diagnosis of lymphoma. The ancillary studies including molecular studies may further improve diagnostic sensitivity and specificity of FNA when needed [14]. The correct diagnosis can be made by FNA expeditiously, and staging procedures and treatment modalities can be instituted right away [1].

\section{Conclusions}

EMPs are very rare entities. These neoplasms are seldom diagnosed by FNAs. All the more, the location of EMP in posterior mediastinum makes it a rather unique case as very few case reports of EMPs arising in posterior mediastinum exist. However, FNA diagnosis of hematopoietic neoplasms is of value not only for the initial diagnosis, but also for the staging of the disease, documentation of recurrence, and identification of tumor transformation [14]. We believe, diagnosis of a posterior mediastinal plasmacytoma by FNA is exceptionally rare and 
Table 1. Locations of Extramedullary Plasmacytomas Diagnosed by FNAs

\begin{tabular}{llll}
\hline NO. & EMP locations & Country & Authors \\
\hline 1 & Gall Bladder & USA & St. Romain et al [4] \\
2 & Liver & Germany & Bangerter et al [1] \\
3 & Tonsil & India & Bhat et al [6] \\
4 & Anterior Mediastinum & USA, Japan, Taiwan & Mallo et al [12] \\
5 & Larynx & USA & Saad et al [9] \\
\hline
\end{tabular}

this report could well be the first of its kind.

\section{Acknowledgments}

We would like to thank the cytotechnologists in the Department of Cytopathology at University of Missouri School of Medicine.

\section{Conflict of Interest}

The authors have no conflict of interest related to this manuscript or in its publication.

\section{Financial Disclosures}

There are no financial disclosures to be made by any of the authors.

\section{References}

1. Bangerter M, Hildebrand A, Waidmann O, Griesshammer M. Fine needle aspiration cytology in extramedullary plasmacytoma. Acta Cytol. 2000;44(3):287-291.

2. Goel G, Rai S, Naik R, Gupta A, Baliga P, Sinha R. Cytodiagnosis of extramedullary plasmacytomas. Acta Cytol. 2010;54(3):255-258.

3. Ganesh M, Sankar NS, Jagannathan R. Extramedullary plasmacytoma presenting as upper back pain. J R Soc Promot Health. 2000;120(4):262-265.

4. St Romain P, Desai S, Bean S, Jiang X, Burbridge RA. Extramedullary plasmacytoma of the gallbladder diagnosed by endoscopic ultrasound fine needle aspiration (EUS-FNA). J Gastrointest Oncol. 2015;6(2):E7-9.

5. Zhang X, Su L, Ran YG, Qie S, Zhang X, Liu C, Shi
HY. Extramedullary plasmacytoma of the trachea: A case report and review of the literature. Medicine (Baltimore). 2018;97(3):e9594.

6. Bhat RV, Prathima K, Harendra Kumar M, Narayana G. Plasmacytoma of tonsil diagnosed by fine-needle aspiration cytology. J Cytol. 2010;27(3):102-103.

7. Sato K, Fumimoto S, Fukada T, Ichihashi Y, Ochi K, Satomi H, Morita T, et al. Extramedullary plasmacytoma arising from the anterior mediastinum. Ann Thorac Surg. 2017;103(5):e393-e395.

8. Hassan MJ, Khans S, Pujani M, Jetley S, Raina PK, Ahmad R. Extramedullary plasmacytoma of the thyroid: report of a rare case. Blood Res. 2014;49(4):280-283.

9. Saad R, Raab S, Liu Y, Pollice P, Silverman JF. Plasmacytoma of the larynx diagnosed by fine-needle aspiration cytology: a case report. Diagn Cytopathol. 2001;24(6):408411.

10. Luh SP, Lai YS, Tsai CH, Tsao TC. Extramedullary plasmacytoma (EMP): Report of a case manifested as a mediastinal mass and multiple pulmonary nodules and review of literature. World J Surg Oncol. 2007;5:123.

11. Lee SY, Kim JH, Shin JS, Shin C, In KH, Kang KH, Yoo $\mathrm{SH}$. A case of extramedullary plasmacytoma arising from the posterior mediastinum. Korean J Intern Med. 2005;20(2):173-176.

12. Mallo R, Gottlieb K, Waggoner D, Wittenkeller J. Mediastinal plasmacytoma detected by echocardiography and biopsied with EUS-FNA. Echocardiography. 2008;25(9):997998.

13. Tani E, Santos GC, Svedmyr E, Skoog L. Fine-needle aspiration cytology and immunocytochemistry of softtissue extramedullary plasma-cell neoplasms. Diagn Cytopathol. 1999;20(3):120-124.

14. Orucevic A, Reddy VB, Selvaggi SM, Green L, Spitz DJ, Gattuso P. Fine-needle aspiration of extranodal and extramedullary hematopoietic malignancies. Diagn Cytopathol. 2000;23(5):318-321. 